\title{
Hot subdwarfs in Resolved Binaries
}

\author{
Simon J. O'Toole
}

(C) Springer-Verlag $\bullet \bullet \bullet \bullet$

\begin{abstract}
In the last decade or so, there have been numerous searches for hot subdwarfs in close binaries. There has been little to no attention paid to wide binaries however. The advantages of understanding these systems can be many. The stars can be assumed to be coeval, which means they have common properties. The distance and metallicity, for example, are both unknown for the subdwarf component, but may be determinable for the secondary, allowing other properties of the subdwarf to be estimated. With this in mind, we have started a search for common proper motion pairs containing a hot subdwarf component. We have uncovered several promising candidate systems, which are presented here.
\end{abstract}

Keywords stars: resolved binaries — stars: hot subdwarfs

\section{Introduction and Motivation}

The mass of a star is of fundamental importance to our understanding of stellar evolution. While there are several robust methods for determining the masses of stars on the Main Sequence, for more evolved non-degenerate stars the situation is much more difficult and many assumptions must be made. Stars at the extreme blue end of the horizontal branch - the hot subdwarfs - fall into this category.

Hot subdwarfs (sdB, sdO) are core helium-burning stars that are found at the blue end of the horizontal branch. They are found in all Galactic stellar populations and are sufficiently numerous to account for the UV-upturn of early-type galaxies. While it is generally accepted they will evolve directly into white dwarfs

Simon J. O'Toole

Anglo-Australian Observatory, PO Box 296, Epping NSW 1710, Australia and not return to the giant phase, exactly how the stars arrived at this point in the HR diagram is still an open question. There are several possible formation channels: binary evolution through mass transfer and common envelope ejection (e.g. Han et al. 2003); delayed core helium flashes in post-Red Giant Branch (RGB) evolution, (e.g. Lanz et al. 2004); the merger of two helium-core white dwarfs (e.g. Saio \& Jeffery 2000); and non-core helium burning post-RGB evolution (e.g. Castellani \& Castellani 1993). The first three channels give rise to a mass distribution peaked near $0.48 M_{\odot}$, while the latter channel produces helium-core white dwarfs with masses closer to $\sim 0.3 M_{\odot}$.

There are only a handful of useful parallax measurements of hot subdwarfs because they were - in general - too faint to be observed with Hipparcos (see Heber et al. 2002). This in turn means they have very few reliable mass determinations. Heber (1992) noted "Hot subluminous stars in binary systems could provide an important tool for checking the evolutionary scenarios ... since they possibly allow stellar masses to be determined. Visual binaries and eclipsing spectroscopic binaries are of utmost importance in this respect." Indeed, about half of the sdBs reside in close binaries with white dwarf or late-type Main Sequence companions. There are several eclipsing spectroscopic binaries containing the latter combination, however these all also show a reflection effect and therefore rely on our incomplete knowledge of the impacts of stellar irradiation (for example, see Drechsel et al. 2001; Vuckovic et al. 2007).

There are some tremendous advantages of studying a hot subdwarf in a resolved binary system. Firstly, the stars do not interact, and most likely have never interacted. This means one does not have to worry about the physics of binary interactions. Secondly, the individual components can be studied independently, without the problems and assumptions surrounding composite spec- 


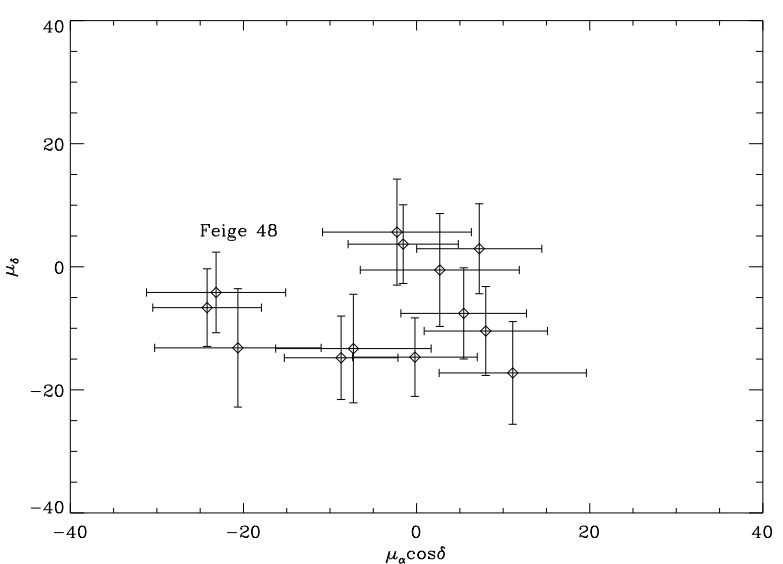

Fig. 1 SuperCosmos proper motions of stars in a field centered on the pulsating sdB Feige 48 (labelled)

tra. Lastly, Main Sequence and Red Giant Branch stars are well understood, at least relative to hot subdwarfs.

We have therefore searched for common proper motion pairs and visual binaries containing a hot subdwarf component. This is the focus of the present work, where we present our most promising candidates and some very preliminary results. The reader should note that there are no definite or clear conclusions in the work presented here; this is an exploratory pilot-type study, which is in part intended to inspire others to explore alternative methods to determining the fundamental parameters of these enigmatic objects.

\section{Visual binaries and Common Proper Motion companions}

With separations large enough to show no spectral contamination, understanding visual binary and common proper motion systems containing hot subdwarf components will be more straightforward than other methods. It allow us to use the spectroscopic parallax to determine the hot subdwarf's mass and metallicity. (Note that while we use the terms "spectroscopic parallax" and "photometric parallax" in this paper, they may be considered as more related to a distance modulus than an angular measurement.) The latter has previously been unknown in field hot subdwarfs (unlike their stellar cluster counterparts), as atmospheric diffusion leads to peculiar abundance patterns (e.g. O'Toole \& Heber 2006).

And while pulsations detected in some sdB stars have allowed the estimation of masses of a handful of objects (e.g. Charpinet et al. 2008), they rely on single-star evolutionary models where a mass range is assumed. Both mass and metallicity are vital in understanding the evolution of both the RGB progenitor and the hot subdwarf itself.

\subsection{Trawling through databases}

As a first step in this project, we have used the Subdwarf Database (Østensen 2006) as the source of known objects. Latter work will use large databases such as the Sloan Digital Sky Survey Data Release 71. After identifying hot subdwarf candidates, we then crosscorrelated their co-ordinates with proper motion and visual binary databases including, but not limited to: The Washington Double Star Catalogue; SuperCosmos; UCAC2; NOMAD1; and Hipparcos. Once pairs are identified, the goal of the project is to determine parameters of companion star and the hot subdwarf.

There are some limitations and difficulties with this approach to be considered. The main concern is that hot subdwarfs are in general quite distant and faint, which means that their proper motions are often very small and/or not well constrained. Figure 1 shows the proper motions for the known binary sdB pulsator Feige 48; the star has a moderately high proper motion $(\sim-25 \mathrm{mas} / \mathrm{yr})$, but cannot be easily distinguished from the other stars in the field around it. In the worst cases though, no proper motions have been measured at all.

Once candidate pairs are selected, we follow two possible avenues to determine their photometric parallax and therefore their distance: empirical relations involving colours (Hawley et al. 2002; Ivezic et al. 2008) or relations using spectral indices (e.g. Cruz \& Reid 2002). The combination of two should lead to a more precise mass estimate. The photometric parallax can then be combined with the spectroscopic parallax to give the mass of the subdwarf. The spectroscopic parallax requires the subdwarf's magnitude, effective temperature and surface gravity. The mass is derived by matching the two parallaxes.

\subsection{The most promising candidates}

From our initial search described above, we have found these promising candidates: BD+481777; FBS 2254+373; PHL 932; and PG1618+563. Thomas Rauch has also discussed the possibility that EC114812303 is also a member of a close pair (these proceedings). Below we discuss the candidates in more detail.

\subsection{1 $B D+481777$}

This bright object is listed in the Luyten Double Star catalogue and has a separation of 127 arcsec from its

\footnotetext{
${ }^{1}$ http://www.sdss.org/dr7/
} 
companion star. The hot subdwarf has a Hipparcos parallax of $6.15 \pm 1.75$ milliarcsseconds (mas), however the reliability of this value is uncertain. The associated uncertainty is too high to meaningfully constrain the star's distance and mass in any case. The corresponding Hipparcos proper motions of $\mu_{\alpha} \cos \delta=83 \pm 2$ mas $/ y r$ and $\mu_{\delta}=-84 \pm 1 \mathrm{mas} / \mathrm{yr}$. The companion star is faint by comparison - it has SDSS photometry, with $(u, g, r, i, z)=(21.074,18.512,17.097,16.138,15.635)-$ and has proper motions of $\mu_{\alpha} \cos \delta=80 \pm 3$ mas $/ \mathrm{yr}$ and $\mu_{\delta}=-83 \pm 3 \mathrm{mas} / \mathrm{yr}$, almost an exact match. The proper motions from SuperCosmos of stars with 150 arcseconds of BD+481777 are shown in Figure 2. The two stars (at $\mu_{\delta} \sim-100$ mas/yr) are clearly separated from the main group around the origin. Note that the SuperCosmos proper motions shown in the figure are somewhat higher than those from the more reliable and precise UCAC2 catalogue quoted above.

$\mathrm{BD}+481777$ is a reasonably well-studied object and we are fortunate to have access to a spectrum, taken using CAFOS on the Calar Alto $2.2 \mathrm{~m}$ with $6 \AA$ resolution and covering wavelength range $3200-6350 \AA$. A model fit of this spectrum kindly carried out by Uli Heber (and shown in Figure 3) using the NLTE model grid of Ströer et al. (2007). The best-fit stellar parameters are given in Table 1. The star is a "garden-variety" helium-rich subdwarf $\mathrm{O}$ star and sits with the majority of these objects in the HR diagram at around $\sim 45000 \mathrm{~K}$ and $\log g \sim 5-6$.

The extinction in the direction of the system is low ( $\sim 0.04 \mathrm{mag})$. The SDSS colours of the companion star are consistent with it being an M0 dwarf. If we use the empirical relations of Hawley et al. (2002) we find a photometric parallax of $\left(i-M_{i}\right) \approx 7.9$ mag. The distance to the star is therefore $\approx 380 \mathrm{pc}$. This in turns leads to the absolute visual magnitude of $\mathrm{BD}+481777$ of $\mathrm{M}_{V} \approx 2.8 \mathrm{mag}$, using $V=10.7$ for the sdO star. We note that these values are very preliminary. Unfortunately the spectrum we have of the He-sdO is not flux calibrated, which makes mass determination for the star currently difficult.

Interestingly, the distance implies that the physical separation is $\approx 48000 \mathrm{AU}$, which is a separation usually found among young binaries. It is expected that those binaries will not survive long before becoming unbound gravitationally.

Table 1 Stellar parameters derived by Uli Heber for the He-sdO BD+48 1777

\begin{tabular}{lc}
\hline$T_{\text {eff }}$ & $44664 \pm 147 \mathrm{~K}$ \\
$\log g$ & $5.84 \pm 3$ \\
$\log (\mathrm{He} / \mathrm{H})$ & $2.69 \pm 0.00$ \\
\hline
\end{tabular}

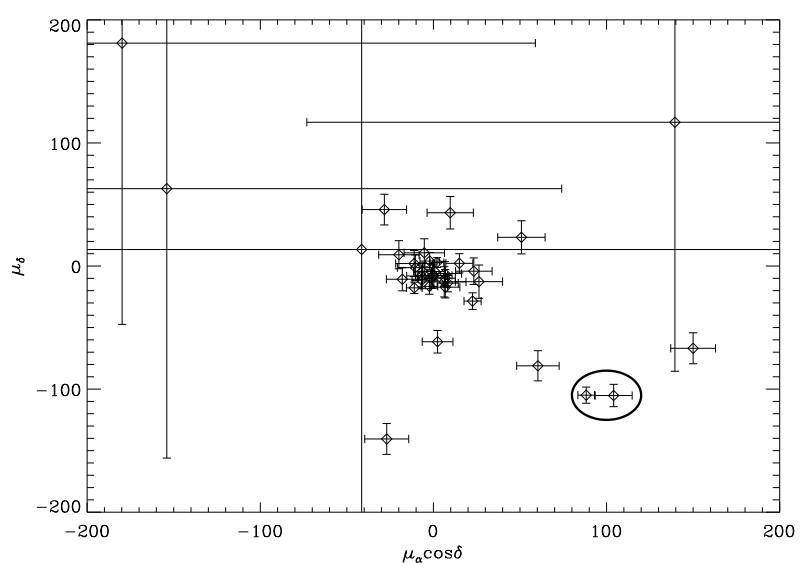

Fig. 2 SuperCosmos proper motions of stars in a field centered on the He-sdO BD+48 1777. The sdO and its companion are circled

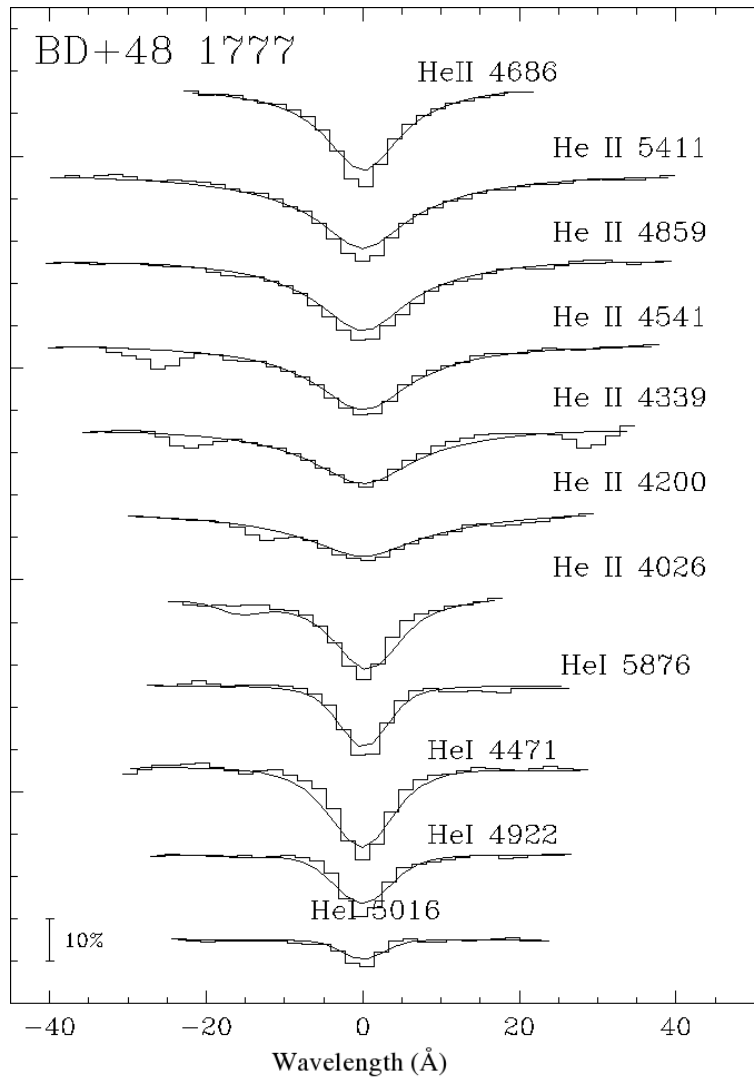

Fig. 3 Model fit of $6 \AA$ spectrum of the He-sdO $\mathrm{BD}+481777$, kindly carried out by Uli Heber 
A spectrum of the M dwarf would lead to better parallax via a spectral index method (e.g. Cruz \& Reid 2002; Reid et al. 1995), while a flux-calibrated spectrum of the He-sdO will lead to the first mass determination of this class of star. The $\mathrm{M}$ dwarf spectrum would also allow the determination of the progenitor system's metallicity, which would be another first for any hot subdwarf. Because it is so bright, this potential makes $\mathrm{BD}+481777$ perhaps the most exciting candidate system we have discovered so far.

\subsubsection{FBS $2254+373$}

This object was classified in the First Byurakan Survey as an sdOD star - in other words, a helium-rich subdwarf. Its proper motion is $145 \mathrm{mas} / \mathrm{yr}$. Unfortunately there is very little good quality photometry of the star and it is relatively faint ( $V \sim 16-17 \mathrm{mag})$, meaning that new photometric measurements are required. The companion star, $\mathrm{BD}+364962$, has the same high proper motion (see Figure 4) and is very bright $(V=10.1 \mathrm{mag})$. Its 2MASS photometry suggests it is a late type star, while its $B-V$ colours (0.99 mag) are consistent with it being a K0 giant star. Somewhat frustratingly, neither star has any published spectra, nor parameter determinations. The separation of the pair is 57 arcsec.

Note that a spectrum of FBS $2254+373$ kindly taken by Thomas Kupfer after this paper was submitted has shown the object to be a DC white dwarf rather than a hot subdwarf star. While this will make it considerably more difficult to determine the mass, it remains a very interesting object.

\subsubsection{PHL 932}

This star, long believed to be the central star of a planetary nebulae (although see Frew. Madsen \& O'Toole (2010) who find that it is not), is the only object in our sample that is well studied spectroscopically and that has a reliable trigonometric parallax $(3.36 \pm 0.62$ mas - Harris et al. 2007). The parallax places the star at a distance of 251-365 pc, but when combined with the spectroscopic parameters from e.g. Lisker et al. (2005), the possible mass range is still large: $0.23-0.5 M_{\odot}$. This means that we cannot distinguish between a star that has undergone core helium-burning (on the EHB) or not (post-RGB). The latter has been assumed by e.g. Napiwotzki (1999) in previous discussion of the object.

The companion to PHL932 has been observed by the SDSS and has the same proper motion ( $\sim 38$ mas/yr from the NOMAD catalogue) and a very wide separation (171 arcsec or $\sim 50,000 \mathrm{AU}$ at the approximate distance to the system). Its SDSS and 2MASS colours are consistent with a spectral type is M2-M3 when compared to Table 3 of Hawley et al. (2002). Using the empirical relation by the same authors, we find $\left(i-M_{i}\right)=7.2 \pm 0.2 \mathrm{mag}$. This constrains the star to a distance in the range $267-302 \mathrm{pc}$, which in turn leads to a mass for PHL 932 of $0.26-0.33 M_{\odot}$. This almost certainly means that the object is not an EHB star, but rather an object that will evolved directly from the RGB to the white dwarf cooling curve, and that will not ignite helium in its core. There are still large uncertainties associated with this determination, however. With a more precise measurement of its photometric parallax, however, we will be able to constrain PHL932's evolutionary history.

\subsection{4 $P G 1618+563$}

This system is also very exciting. It consists of a pulsating sdB star and an F3V star in a close $(\sim 3$ arcsec $)$ binary. Silvotti et al. (2000) discovered the pulsations in the sdB, and discussed the companion's spectral type and status, however did not go any further. Both stars are relatively bright (sdB: 13.3, F3: 12.8), so a detailed study of this system is possible. With commonly used analysis methods, the distance, metallicity and age of the F3V star should be straightforward to measure, which in turn will lead to the mass of the sdB star, independent of asteroseismological models. This system will provide an excellent test of both evolution and pulsation models for sdB stars.

\section{Future Work}

We have presented the first results and motivation for an exciting new method to determine the mass of hot subdwarf stars independent of evolutionary models, which necessarily assume an atmospheric structure and mass range.

There are several questions surrounding the systems presented here that warrant further investigation. Some of these stars have very wide physical separations; how could they stay gravitationally bound for so long? Could they instead be two stars that were once in a closer binary system and were then "ejected" after a mass-transfer phase? Can we even describe them as binary systems or are they more similar to co-moving groups, despite their age?

We need a large study of sdB proper motions using archival and possibly new data. With the release of the UCAC3 catalogue, which has larger sky coverage and higher precision proper motions (at least in some 
cases) some progress is already being made in this direction. The rise of survey telescopes (e.g. SkyMapper) may make it more and more straightforward to obtain accurate proper motions in the coming decade. On top of these surveys, the GAIA space mission will deliver precise astrometry (to $\sim 0.3$ mas) of stars down to $\mathrm{V} \approx 20 \mathrm{mag}$. Since space velocities are in three dimensions, it will also be important to obtain relatively precise radial velocities, allowing the status of the candidates presented here to be confirmed.

We plan to carry out a detailed spectroscopic study of the companion stars presented here, as well as several other candidates found in the Sloan Digital Sky Survey database. We will use spectra to determine photometric parallaxes of the late $\mathrm{K}$ and $\mathrm{M}$ dwarf companions by measuring $\mathrm{TiO}-, \mathrm{CaH}-$ and $\mathrm{CaOH}$-band spectral indices using the prescription of Cruz \& Reid (2002) and metallicities using the calibration of Woolf \& Wallerstein (2006). We also plan to observe hot subdwarfs with no available spectroscopy, and then measure their effective temperatures, surface gravities and helium abundances using standard line-profile fitting (e.g. Lisker et al. 2005; Ströer et al. 2007).

There are several late G-/early K-type companions among our sample (not discussed here) and we will initally use the empirical relationships of Ivezic et al. (2008), before eventually obtaining high resolution spectra and conducting a more detailed analysis of their parameters.

The successful completion of this project will dramatically increase the number of hot subdwarfs with accurate mass determinations independent of evolution models. We will also determine the first accurate masses for helium-rich subdwarfs, which is a class of stars that cannot be investigated using other methods, such as stellar oscillations and asteroseismology. The masses

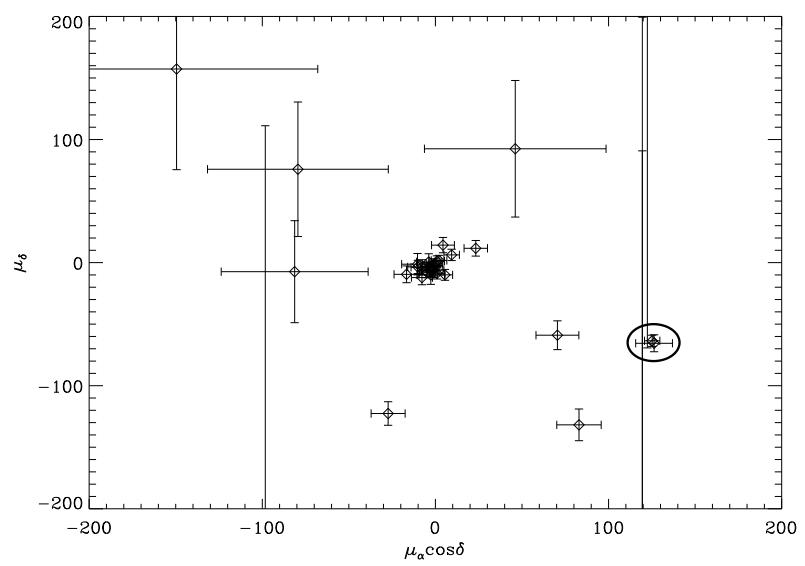

Fig. 4 SuperCosmos proper motions of stars in a field centered on FBS $2254+373$. The star and its companion are circled

will allow the first robust tests of the proposed formation channels for some of these objects (e.g. Justham, these proceedings). 


\section{References}

Castellani \& Castellani, 1993, ApJ, 407, 649

Charpinet, S. et al. 2008, A\&A, 489, 377

Cruz, K. \& Reid, I. N. 2002, AJ, 123, 2828

Drechsel, H. et al. 2001, A\&A, 378, 893

Frew, D., Madsen, G. \& O'Toole, S. J., 2010, PASA, in press, astro-ph/0910.2078

Han, Z. et al. 2003, MNRAS, 341, 669

Harris, H. et al. 2007, AJ, 133, 631

Hawley, S. et al. 2002, AJ, 123, 3409

Heber, U., 1992, Lectures in Physics, 401, 233

Heber, U., et al. 2002, A\&A, 383, 938

Ivezic et al., 2008, ApJ, 684, 287

Lisker, T., et al. 2005, A\&A, 430, 223

Lanz, T. et al. 2004, ApJ, 602, 342

Napiwotzki, R., 1999, A\&A, 350, 101

Østensen, R., 2006, Baltic Astronomy

O'Toole, S. J. \& Heber, U., 2006, A\&A, 452, 579

Reid, I. N. et al. 1995, AJ, 110, 1838

Saio, H. \& Jeffery, C. S. 2000, MNRAS, 313, 671

Silvotti, R., et al. 2000, A\&A, 359, 1068

Ströer, A., et al. 2007, A\&A, 462, 269

Vuckovic, M. et al. 2007, A\&A, 471, 605

Woolf, V. M. \& Wallerstein, G., 2006, PASP, 118, 218 